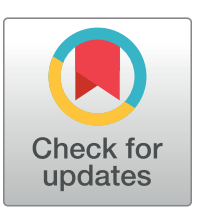

fopen access

Citation: Webb BJ, Levin NM, Grisel N, Brown SM, Peltan ID, Spivak ES, et al. (2022) Simple scoring tool to estimate risk of hospitalization and mortality in ambulatory and emergency department patients with COVID-19. PLoS ONE 17(3): e0261508. https://doi.org/10.1371/journal.pone.0261508

Editor: Carlo Torti, University "Magna Graecia" of Catanzaro, ITALY

Received: February 23, 2021

Accepted: December 4, 2021

Published: March 3, 2022

Peer Review History: PLOS recognizes the benefits of transparency in the peer review process; therefore, we enable the publication of all of the content of peer review and author responses alongside final, published articles. The editorial history of this article is available here: https://doi.org/10.1371/journal.pone.0261508

Copyright: $\odot 2022$ Webb et al. This is an open access article distributed under the terms of the Creative Commons Attribution License, which permits unrestricted use, distribution, and reproduction in any medium, provided the original author and source are credited.

Data Availability Statement: In order to protect patient privacy and comply with institutional data use policy, data used in this study are unavailable to upload to public servers. As required by the
RESEARCH ARTICLE

\section{Simple scoring tool to estimate risk of hospitalization and mortality in ambulatory and emergency department patients with COVID-19}

\author{
Brandon J. Webb ${ }^{1,2} *$, Nicholas M. Levin ${ }^{3}{ }^{3}$, Nancy Grisel ${ }^{4}$, Samuel M. Brown ${ }^{5}$, Ithan \\ D. Peltan ${ }^{5}$, Emily S. Spivak ${ }^{6}$, Mark Shah ${ }^{7}$, Eddie Stenehjem ${ }^{1,2,8}$, Joseph Bledsoe ${ }^{7,9}$
}

1 Division of Infectious Diseases and Clinical Epidemiology, Intermountain Healthcare, Salt Lake City, UT, United States of America, 2 Division of Infectious Diseases and Geographic Medicine, Stanford Medicine, Palo Alto, CA, United States of America, 3 Division of Emergency Medicine, University of Utah School of Medicine, Salt Lake City, UT, United States of America, 4 Intermountain Healthcare, Enterprise Analytics, Salt Lake City, UT, United States of America, 5 Division of Pulmonary and Critical Care Medicine, Intermountain Medical Center and University of Utah, Salt Lake City, UT, United States of America, 6 Division of Infectious Diseases, University of Utah School of Medicine, Salt Lake City, UT, United States of America, 7 Intermountain Healthcare, Department of Emergency Medicine, Salt Lake City, UT, United States of America, 8 Intermountain Healthcare, Office of Patient Experience, Salt Lake City, UT, United States of America, 9 Stanford Medicine, Department of Emergency Medicine, Palo Alto, CA, United States of America

* Brandon.Webb@imail.org

\section{Abstract}

\section{Background}

Accurate methods of identifying patients with COVID-19 who are at high risk of poor outcomes has become especially important with the advent of limited-availability therapies such as monoclonal antibodies. Here we describe development and validation of a simple but accurate scoring tool to classify risk of hospitalization and mortality.

\section{Methods}

All consecutive patients testing positive for SARS-CoV-2 from March 25-October 1, 2020 within the Intermountain Healthcare system were included. The cohort was randomly divided into $70 \%$ derivation and $30 \%$ validation cohorts. A multivariable logistic regression model was fitted for 14-day hospitalization. The optimal model was then adapted to a simple, probabilistic score and applied to the validation cohort and evaluated for prediction of hospitalization and 28-day mortality.

\section{Results}

22,816 patients were included; mean age was 40 years, $50.1 \%$ were female and $44 \%$ identified as non-white race or Hispanic/Latinx ethnicity. $6.2 \%$ required hospitalization and $0.4 \%$ died. Criteria in the simple model included: age ( 0.5 points per decade); high-risk comorbidities ( 2 points each): diabetes mellitus, severe immunocompromised status and obesity (body mass index $\geq 30$ ); non-white race/Hispanic or Latinx ethnicity (2 points), and 1 point 
Intermountain Healthcare Institutional Review Board, data sharing agreement requests to access deidentified versions of the datasets generated and/ or analyzed during the current study may be addressed to the Intermountain Office of Research (officeofresearch@imail.org).

Funding: IP reports salary support through a grant from the National Institutes of Health (U.S.A). SB reports salary support from the U.S. NIH, Centers for Disease Control and the Department of Defense; he also reports receiving support for chairing a data and safety monitoring board for a respiratory failure trial sponsored by Hamilton, effort paid to Intermountain for steering committee work for Faron Pharmaceuticals and Sedana Pharmaceuticals for ARDS work, support from Janssen for Influenza research, and royalties for books on religion and ethics from 0xford University Press/Brigham Young University. BW reports partial salary support from a U.S. Federal grant from AHRQ. ES receives partial salary support through grants from the Centers for Disease Control. At the time of submission, Intermountain Healthcare and the University of Utah have participated in COVID-19 trials sponsored by: Abbvie, Genentech, Gilead, Regeneron, Roche, and the U.S. National Institutes of Health ACTIV and PETAL clinical trials networks; several authors (BW, IP, JB, SB, ES) were site investigators on these trials but received no direct or indirect remuneration for their effort. ES, BJW, SMB and MS are members of the Utah crisis standards of care scarce medication committee.

Competing interests: IP reports salary support through a grant from the National Institutes of Health (U.S.A). SB reports salary support from the U.S. NIH, Centers for Disease Control and the Department of Defense; he also reports receiving support for chairing a data and safety monitoring board for a respiratory failure trial sponsored by Hamilton, effort paid to Intermountain for steering committee work for Faron Pharmaceuticals and Sedana Pharmaceuticals for ARDS work, support from Janssen for Influenza research, and royalties for books on religion and ethics from 0xford University Press/Brigham Young University. BW reports partial salary support from a U.S. Federal grant from $A H R Q$. ES receives partial salary support through grants from the Centers for Disease Control. At the time of submission, Intermountain Healthcare and the University of Utah have participated in COVID-19 trials sponsored by: Abbvie, Genentech, Gilead, Regeneron, Roche, and the U.S. National Institutes of Health ACTIV and PETAL clinical trials networks; several authors (BW, IP, JB, SB, ES) were site each for: male sex, dyspnea, hypertension, coronary artery disease, cardiac arrythmia, congestive heart failure, chronic kidney disease, chronic pulmonary disease, chronic liver disease, cerebrovascular disease, and chronic neurologic disease. In the derivation cohort $(n=16,030)$ area under the receiver-operator characteristic curve (AUROC) was $0.82(95 \%$ $\mathrm{Cl} 0.81-0.84)$ for hospitalization and $0.91(0.83-0.94)$ for 28-day mortality; in the validation cohort $(n=6,786)$ AUROC for hospitalization was $0.8(\mathrm{Cl} 0.78-0.82)$ and for mortality 0.8 (Cl 0.69-0.9).

\section{Conclusion}

A prediction score based on widely available patient attributes accurately risk stratifies patients with COVID-19 at the time of testing. Applications include patient selection for therapies targeted at preventing disease progression in non-hospitalized patients, including monoclonal antibodies. External validation in independent healthcare environments is needed.

\section{Introduction}

COVID-19 is a systemic infection caused by a novel betacoronavirus, SARS-COV-2 [1]. A relatively conserved set of clinical and demographic factors are now recognized to correlate with an increased risk for severe disease requiring hospitalization, mechanical ventilation and death [2-4]. Accurate methods of risk stratifying ambulatory patients at the point of test positivity has many possible applications, including prioritizing patients at highest risk of hospitalization for early treatments aimed to prevent progression to severe disease, such as monoclonal antibodies, which are both limited in availability and also more likely to be effective in high-risk groups. Several models have been proposed [3,5-9]. We describe development and validation of a simple scoring model to predict hospitalization and mortality in a large cohort of ED and ambulatory patients with COVID-19.

\section{Methods}

Intermountain Healthcare is an integrated healthcare system that provides care to more than 1.5 million patients each year in Utah and bordering communities. As part of a systemwide COVID-19 response, Intermountain provides SARS-CoV-2 testing at 32 urgent care facilities, 23 emergency departments, and 16 community drive-up testing sites. During the study period, only polymerase chain reaction (PCR) assays were performed (Thermofisher, Waltham, MA; Cepheid, Sunnyvale, CA; Quidel, San Diego, CA, BioFire, Salt Lake City, UT; Roche, Basel, Switzerland). All testing required an order entered in the electronic health record (EHR) (Cerner, Kansas City, KS) by the ordering clinician through a structured form that requires the clinician to input the patient's clinical symptoms and epidemiological features. These data are stored in the Intermountain Prospective Observational COVID-19 (IPOC) database, and the enterprise data warehouse.

We queried the IPOC database for consecutive adult patients with positive SARS-CoV-2 tests from March 25-October 1, 2020. Symptom data were extracted from the electronic test order form while demographic and co-morbidity data were obtained from the IPOC database and data warehouse using the Charlson and Elixhauser definitions $[10,11]$. We defined immunosuppression as: recipient of a solid organ or hematopoietic stem cell transplant, on 
investigators on these trials but received no direct or indirect remuneration for their effort. ES, BJW, SMB and MS are members of the Utah crisis standards of care scarce medication committee. This does not alter our adherence to PLOS ONE policies on sharing data and materials. At the time of submission, Intermountain Healthcare and the University of Utah have participated in COVID-19 trials sponsored by: Abbvie, Genentech, Gilead, Regeneron, Roche, and the U.S. National Institutes of Health ACTIV and PETAL clinical trials networks; several authors (BW, IP, JB, SB, ES) were site investigators on these trials but received no direct or indirect remuneration for their effort. ES, BJW, SMB and MS are members of the Utah crisis standards of care scarce medication committee. chemotherapy, biologic or other immunosuppressive agents targeting B or T cell activity, chronic corticosteroids at a prednisone-equivalent dose of $20 \mathrm{mg}$ per day or greater for more than 30 days, human immunodeficiency virus complicated by acquired immunodeficiency syndrome (AIDS), heritable immunodeficiency. We defined obesity as body mass index (BMI) of greater than or equal to 30 [12]. Symptom and demographic data were complete; comorbidity data were complete insofar as patients had prior encounters in the integrated health system. Mortality data was captured via an existing linkage to state death records.

We used a random number generator to divide the cohort into a 70\% derivation cohort and $30 \%$ validation cohort. In the derivation cohort data, we fitted a multivariable logistic regression model for hospitalization within 14 days of testing, using clinical and demographic features. Predictors were prespecified before model development based on: clinical features that would be available at the time of testing for all ambulatory and emergency department patients regardless of testing venue (a criterion that precludes, for instance, laboratory data), biological plausibility of association with severity, and reproducibility in other studies in existing COVID-19 literature. We intentionally did not fit a model for mortality, but instead planned $a$ priori to validate the ultimate model against that outcome. Model discrimination was evaluated using the area under the receiver-operator characteristic curve (AUROC) and model fit by evaluating $\mathrm{R}^{2}$ using the Nagelkerke method because the Hosmer-Lemeshow goodness-of-fit is not valid in very large sample sizes $[13,14]$.

We included patients who tested in the ambulatory setting as well as patients who tested positive in the emergency department to ensure that the score would be applicable in both environments. However, we recognized that some patients testing positive in the emergency department (ED) are then subsequently admitted. The decision to admit or not is not immediately known to emergency medicine providers who may still wish to use the score to stratify risk to aid in clinical decision making and selection of therapies. However, because patients who are admitted from the ED may have different characteristics than those tested in the ambulatory setting, we planned a priori to perform a sensitivity analysis by repeating the regression above after restricting the cohort to patients who were not admitted to the hospitalization at the time of their test.

We then adapted the original logistic regression model into a simple scoring tool by converting exponentiated $\beta$ coefficients into weighted point assignments for each variable. We evaluated the test performance characteristics of this simplified clinical prediction tool in the derivation and validation cohorts using AUROC and by calculating the sensitivity, specificity, negative and positive predictive values across the range of scoring thresholds. To account for possible secular changes in patient distribution or variant epidemiology, we performed a temporally-independent internal validation of the scoring tool in a cohort comprised of all laboratory-confirmed COVID-19 patients in the IPOC database from November 1, 2020 to August $15,2021$.

This study was approved by the Intermountain Healthcare Institutional Review Board which granted a waiver of informed consent to use patient data collected and stored for public health purposes.

\section{Results}

From March 25 through October 1, 2020, 22,816 patients had a positive PCR test for SARS-CoV-2. The mean age was 40 years (see Table 1); 11,424 (50.1\%) patients were female and $8753(43.9 \%)$ identified as a member of a community of color (either non-white race or Hispanic or Latinx ethnicity). Patients had on average one significant medical comorbidity. 1419 (6.2\%) of patients were admitted; of these, 799 (3.6\%) tested positive in the emergency 
Table 1. Patient characteristics, total and by derivation and validation cohort groups.

\begin{tabular}{|c|c|c|c|}
\hline & ALL & DERIVATION & VALIDATION \\
\hline & N (\%) unless noted & N (\%) unless noted & N (\%) unless noted \\
\hline All Patients & $22816(100)$ & $16030(70.3)$ & $6786(29.7)$ \\
\hline Male & $11392(49.9)$ & $8005(49.9$ & $3387(49.9)$ \\
\hline Age, years (Mean, SD) & $40.4(16.5)$ & $40.4(16.5)$ & $40.2(16.6)$ \\
\hline \multicolumn{4}{|l|}{ Race } \\
\hline American Indian or Alaska Native & $238(1.0)$ & $169(1.1)$ & $69(1.0)$ \\
\hline Asian & $349(1.5)$ & $238(1.5)$ & $111(1.6)$ \\
\hline Black or African American & $341(1.5)$ & $233(1.5)$ & $108(1.6)$ \\
\hline Multiple & $78(0.3)$ & $56(0.3)$ & $22(0.3)$ \\
\hline Native Hawaiian or Pacific Islander & $893(3.9)$ & $626(3.9)$ & $267(3.9)$ \\
\hline White & $16624(72.9)$ & $11637(72.6)$ & $4987(73.5)$ \\
\hline \multicolumn{4}{|l|}{ Ethnicity } \\
\hline Hispanic, Latino, or Spanish origin & $7027(30.8)$ & $4980(31.1)$ & $2047(30.2)$ \\
\hline Communities of Color ${ }^{1}$ & $8753(43.9)$ & $6184(44.3)$ & $2569(43.0)$ \\
\hline \multicolumn{4}{|l|}{ Symptoms (Reported at time of test) } \\
\hline Fever & $7889(34.6)$ & $5561(34.7)$ & $2328(34.3)$ \\
\hline Cough & $11595(50.8)$ & $8188(51.1)$ & $3407(50.2)$ \\
\hline Dyspnea & $6008(26.3)$ & $4273(26.7)$ & $1735(25.6)$ \\
\hline Myalgia & $11341(49.7)$ & $7985(49.8)$ & $3356(49.5)$ \\
\hline Rhinorrhea & $8843(38.8)$ & $6203(38.7)$ & $2640(38.9)$ \\
\hline Anosmia & $5164(22.6)$ & $3681(23.0)$ & $1483(21.9)$ \\
\hline Pharyngitis & $8130(35.6)$ & $5718(35.7)$ & $2412(35.5)$ \\
\hline Diarrhea & $3648(16.0)$ & $2573(16.1)$ & $1075(15.8)$ \\
\hline \multicolumn{4}{|l|}{ Comorbidities } \\
\hline Count, Mean (SD), Range & 0.7 (1.3), $0-11$ & 0.7 (1.3), $0-11$ & $0.7(1.3), 0-10$ \\
\hline Diabetes Mellitus & $2164(9.5)$ & $1532(9.6)$ & $632(9.3)$ \\
\hline Hypertension & $3897(17.1)$ & $2816(17.6)$ & $1081(15.9)$ \\
\hline Cardiovascular Disease & $331(1.5)$ & $246(1.5)$ & $85(1.3)$ \\
\hline Cardiac Arrhythmia & $2437(10.7)$ & $1704(10.6)$ & $733(10.8)$ \\
\hline Chronic Pulmonary Disease & $4231(18.5)$ & $2920(18.2)$ & $1311(19.3)$ \\
\hline Chronic Kidney Disease & $687(3.0)$ & $507(3.2)$ & $180(2.7)$ \\
\hline Congestive Heart Failure & $536(2.3)$ & $384(2.4)$ & $152(2.2)$ \\
\hline Chronic Liver Disease & $1320(5.8)$ & $914(5.7)$ & $406(6.0)$ \\
\hline Obesity & $3395(14.9)$ & $2376(14.8)$ & $1019(15.0)$ \\
\hline Immunosuppression & $143(0.6)$ & $101(0.6)$ & $42(0.6)$ \\
\hline Cerebrovascular Disease & $589(2.6)$ & $409(2.6)$ & $180(2.7)$ \\
\hline Neurological Disorders & $1037(4.5)$ & $723(4.5)$ & $314(4.6)$ \\
\hline History of Tobacco Use & $3324(21.5)$ & $2295(21.2)$ & $1029(22.1)$ \\
\hline Mortality, 28-Day All-Cause & $93(0.4)$ & $73(0.5)$ & $20(0.3)$ \\
\hline Hospitalization, 14-Day & $1419(6.2)$ & $990(6.2)$ & $429(6.3)$ \\
\hline
\end{tabular}

Abbreviations: SE: Standard Error.

${ }^{1}$ Self-identifies as either non-white race or Hispanic/Latinx ethnicity. 
Table 2. Demographics and clinical characteristics of the temporally-independent validation cohort.

\begin{tabular}{|c|c|c|}
\hline & \multicolumn{2}{|c|}{$\begin{array}{l}\text { Laboratory-confirmed COVID-19 Positive } \\
\text { Patients }\end{array}$} \\
\hline & $\mathbf{N}^{*}$ & $(\%)^{*}$ \\
\hline Total, N (\%) & 86,130 & \\
\hline \multicolumn{3}{|l|}{ Demographics } \\
\hline Age, mean years (SD) & 42.5 & 16.9 \\
\hline Female & 44892 & 52.1 \\
\hline Race, American Indian or Alaskan Native & 662 & 0.8 \\
\hline Race, Asian & 1072 & 1.2 \\
\hline Race, Black or African American & 773 & 0.9 \\
\hline Race, Native Hawaiian or Pacific Islander & 1416 & 1.6 \\
\hline Race, White & 87525 & 82.2 \\
\hline Race, other or multiple & 11378 & 13.2 \\
\hline Hispanic or Latinx Ethnicity & 10859 & 12.6 \\
\hline Community of Color & 23575 & 27.4 \\
\hline \multicolumn{3}{|l|}{ Symptoms (at time of positive test) } \\
\hline Fever & 26197 & 30.4 \\
\hline Cough & 45828 & 53.2 \\
\hline Shortness of breath & 19251 & 22.4 \\
\hline Myalgia & 44955 & 52.2 \\
\hline Rhinorrhea & 38770 & 45.0 \\
\hline Altered sense of smell & 18462 & 21.4 \\
\hline Pharyngitis & 33095 & 38.4 \\
\hline Diarrhea & 11886 & 13.8 \\
\hline \multicolumn{3}{|l|}{ Comorbidities } \\
\hline Total Comorbidities, median (IQI) & 0 & $0-1$ \\
\hline Immunocompromised status & 603 & 0.7 \\
\hline Diabetes Mellitus & 7186 & 8.3 \\
\hline Coronary Artery Disease & 1408 & 1.6 \\
\hline Active Malignancy & 560 & 0.7 \\
\hline Chronic Pulmonary Disease & 19758 & 22.9 \\
\hline Chronic Kidney Disease & 2976 & 3.5 \\
\hline Chronic Liver Disease & 5484 & 6.4 \\
\hline Cerebrovascular Disease & 2697 & 3.1 \\
\hline Hypertension & 16724 & 19.4 \\
\hline Chronic Neurological Disease & 4164 & 4.8 \\
\hline Congestive Heart Failure & 2229 & 2.6 \\
\hline Cardiac Arrhythmia & 10701 & 12.4 \\
\hline Obesity & 14486 & 16.8 \\
\hline \multicolumn{3}{|l|}{ Outcomes } \\
\hline Hospitalization within 14 days & 2555 & 3.0 \\
\hline Mortality within 28 days & 293 & 0.3 \\
\hline
\end{tabular}

https://doi.org/10.1371/journal.pone.0261508.t002

In the derivation cohort, clinical features by hospitalization status are reported in Table 3. The primary multivariable model (see Table 4 ) demonstrated adequate model diagnostics [AUROC 0.824 (95\% CI 0.809-0.840), Nagelkerke $\mathrm{R}^{2} 0.26$ ]. Age, male sex, self-identification to a community of color, dyspnea and high-risk comorbidities including diabetes mellitus, obesity, immunosuppression and chronic neurologic disease were each associated with 
Table 3. Patient characteristics of the derivation cohort stratified by outcome of hospitalization.

\begin{tabular}{|c|c|c|}
\hline & \multicolumn{2}{|c|}{ Hospitalized } \\
\hline & No & Yes \\
\hline & $\mathrm{N}(\%)$ unless noted & $\mathrm{N}(\%)$ unless noted \\
\hline $\mathbf{N}=$ & 15040 & 990 \\
\hline Male & $7472(49.7 \%)$ & $533(53.8)$ \\
\hline Age, Years (Mean, SD) & $39.5(16)$ & $54.8(17.7)$ \\
\hline \multicolumn{3}{|l|}{$\underline{\text { Race }}$} \\
\hline American Indian or Alaska Native & $145(1.0)$ & $24(2.4)$ \\
\hline Asian & $218(1.4)$ & $20(2.0)$ \\
\hline Black or African American & $217(1.4)$ & $16(1.6)$ \\
\hline Multiple & $55(0.4)$ & $1(0.1)$ \\
\hline Native Hawaiian or Pacific Islander & $524(3.5)$ & $102(10.3)$ \\
\hline White & $10940(72.7)$ & $697(70.4)$ \\
\hline \multicolumn{3}{|l|}{ Ethnicity } \\
\hline Hispanic or Latinx & $4622(30.7)$ & $358(36.2)$ \\
\hline Communities of Color ${ }^{1}$ & $5671(43.5)$ & $513(54.7)$ \\
\hline \multicolumn{3}{|l|}{ Symptoms (at time of testing) } \\
\hline Fever & $4999(33.2)$ & $562(56.8)$ \\
\hline Cough & $7575(50.4)$ & $613(61.9)$ \\
\hline Dyspnea & $3707(24.6)$ & $566(57.2)$ \\
\hline Myalgia & $7462(49.6)$ & $523(52.8)$ \\
\hline Rhinorrhea & $5961(39.6)$ & $242(24.4)$ \\
\hline Anosmia & $3516(23.4)$ & $165(16.7)$ \\
\hline Pharyngitis & $5476(36.4)$ & $242(24.4)$ \\
\hline Diarrhea & $2379(15.8)$ & $194(19.6)$ \\
\hline \multicolumn{3}{|l|}{ Comorbidities } \\
\hline Comorbidity Count, (Mean, SD), Range & $0.7(1.2), 0-10$ & $2.1(2.0) 0-11$ \\
\hline Diabetes Mellitus & $1145(7.6)$ & $387(39.1)$ \\
\hline Hypertension & $2308(15.3)$ & $508(51.3)$ \\
\hline Cardiovascular Disease & $178(1.2)$ & $68(6.9)$ \\
\hline Cardiac Arrhythmia & $1442(9.6)$ & $262(26.5)$ \\
\hline Chronic Pulmonary Disease & $2620(17.4)$ & $300(30.3)$ \\
\hline Chronic Kidney Disease & $357(2.4)$ & $150(15.2)$ \\
\hline Congestive Heart Failure & $261(1.7)$ & $123(12.4)$ \\
\hline Chronic Liver Disease & $768(5.1)$ & $146(14.7)$ \\
\hline Obesity & $1987(13.2)$ & $389(39.3)$ \\
\hline Immunosuppression & $82(0.5)$ & $19(1.9)$ \\
\hline Cerebrovascular Disease & $315(2.1)$ & $94(9.5)$ \\
\hline Chronic Neurological Disease & $573(3.8)$ & $150(15.2)$ \\
\hline History of Tobacco Use & $2012(20.5)$ & $283(28.6)$ \\
\hline Mortality, 28-Day All-Cause & $13(0.1)$ & $60(6.1)$ \\
\hline
\end{tabular}

Abbreviations: SE: Standard Error.

${ }^{1}$ Self-identifies as either non-white race or Hispanic/Latinx ethnicity.

https://doi.org/10.1371/journal.pone.0261508.t003

significantly greater odds of hospitalization. In an exploratory analysis in which individual comorbidities were replaced in the regression with a count of total comorbidities, the cumulative number of comorbidities was also significant (OR 1.4, 95\% CI 1.3-1.5). In the planned sensitivity analysis excluding patients who were tested in the emergency department during 
Table 4. Multivariable logistic regression model for hospitalization in the derivation cohort.

\begin{tabular}{l|c|c|c}
\hline & $\mathbf{p}$ & Adjusted Odds Ratio & 95\% CI \\
\hline Age (decades) & $<0.0001$ & 1.5 & $1.4-1.6$ \\
\hline Male & $<0.0001$ & 2.1 & $1.2-1.6$ \\
\hline Communities of color ${ }^{1}$ & $<0.0001$ & 3.5 & $1.8-2.4$ \\
\hline Dyspnea & $<0.0001$ & 2.2 & $1.0-4.0$ \\
\hline Diabetes mellitus & $<0.0001$ & 0.88 & $1.8-2.6$ \\
\hline Hypertension & 0.001 & 1.1 & $0.1-1.7$ \\
\hline Coronary Artery Disease & 0.45 & 0.92 & $0.9-1.3$ \\
\hline Cardiac Arrhythmia & 0.41 & 1.1 & $0.8-1.1$ \\
\hline Chronic Pulmonary Disease & 0.39 & 1.3 & $0.9-1.5$ \\
\hline Chronic Kidney Disease & 0.29 & 1.0 & $1.0-1.8$ \\
\hline Congestive Heart Failure & 0.07 & 1.9 & $0.8-1.2$ \\
\hline Chronic Liver Disease & 0.98 & 3.9 & $1.6-2.3$ \\
\hline Obesity & $<0.0001$ & 1.1 & $1.3-12.1$ \\
\hline Immunosuppression & 2 & 1.8 & $0.8-1.4$ \\
\hline Cerebrovascular Disease & 0.02 & & $1.4-2.4$ \\
\hline Chronic Neurologic Disease & 0.74 & & \\
\hline
\end{tabular}

${ }^{1}$ Self-identifies as either non-white race or Hispanic/Latinx ethnicity.

${ }^{2}$ Excludes patients with metastatic cancer with non-hospitalization goals of care.

https://doi.org/10.1371/journal.pone.0261508.t004

their admission to the hospital, the multivariable model had slightly diminished performance [AUROC 0.789 (95\% CI: 0.768-0.810), $\mathrm{R}^{2}$ 0.164]. Overall, contributions of individual risk factors were similar in this model compared to the model including patients being admitted, (see Table 5) with the exception that the magnitude of risk of dyspnea was less in the ambulatory-

Table 5. Sensitivity Analysis: Multivariable logistic regression model for hospitalization in the derivation cohort, excluding patients admitted from the emergency department.

\begin{tabular}{l|c|c|c}
\hline & $\mathbf{p}$ & Adjusted Odds Ratio & 95\% CI \\
\hline Age (decades) & $<0.0001$ & 1.5 & $1.4-1.6$ \\
\hline Male & 0.003 & 1.3 & $1.1-1.6$ \\
\hline Communities of color ${ }^{1}$ & $<0.0001$ & 1.8 & $1.5-2.2$ \\
\hline Dyspnea & $<0.0001$ & 2.1 & $1.7-2.5$ \\
\hline Diabetes mellitus & $<0.0001$ & 2.1 & $1.6-2.6$ \\
\hline Hypertension & 0.001 & 1.2 & $1.0-1.6$ \\
\hline Coronary Artery Disease & 0.91 & 1.0 & $0.6-1.5$ \\
\hline Cardiac Arrhythmia & 0.39 & 1.1 & $0.9-1.4$ \\
\hline Chronic Pulmonary Disease & 0.12 & 1.2 & $1.0-1.4$ \\
\hline Chronic Kidney Disease & 0.89 & 1.0 & $0.7-1.4$ \\
\hline Congestive Heart Failure & 0.72 & 1.1 & $0.7-1.6$ \\
\hline Chronic Liver Disease & 0.87 & 1.0 & $0.8-1.4$ \\
\hline Obesity & $<0.0001$ & 1.8 & $1.5-2.3$ \\
\hline Immunosuppression & & & \\
\hline Cerebrovascular Disease & 0.003 & 7.0 & $2.0-24.9$ \\
\hline Chronic Neurologic Disease & 0.25 & 1.2 & $0.7-1.5$ \\
\hline
\end{tabular}

${ }^{1}$ Self-identifies as either non-white race or Hispanic/Latinx ethnicity.

${ }^{2}$ Excludes patients with metastatic cancer with non-hospitalization goals of care.

https://doi.org/10.1371/journal.pone.0261508.t005 
Table 6. Simplified clinical prediction score for COVID-19 outcomes.

\begin{tabular}{|c|c|}
\hline Demographic Risk Factors & Points \\
\hline Male & 1 \\
\hline Age & $\begin{array}{c}\mathbf{0 . 5} \text { for every decade: } \\
0-10=\mathbf{0 . 5}, 11-20=\mathbf{1}, 21-30=\mathbf{1 . 5}, 31-40=\mathbf{2}, 41-50=\mathbf{2 . 5}, 51-60=\mathbf{3}, 61-70=\mathbf{3 . 5}, \\
71-80=\mathbf{4}, 81-90=\mathbf{4 . 5}, 91-100=\mathbf{5},>100=\mathbf{5 . 5}\end{array}$ \\
\hline Communities of color ${ }^{1}$ & 2 \\
\hline \multicolumn{2}{|l|}{ High Risk Comorbidities } \\
\hline Diabetes Mellitus & 2 \\
\hline $\begin{array}{l}\text { Severely } \\
\text { Immunocompromised }^{2}\end{array}$ & 2 \\
\hline Obesity (BMI > 30) & 2 \\
\hline \multicolumn{2}{|l|}{ Other Comorbidities } \\
\hline Hypertension & 1 \\
\hline Coronary Artery Disease & 1 \\
\hline Cardiac Arrhythmia & 1 \\
\hline Congestive Heart Failure & 1 \\
\hline Chronic Kidney Disease & 1 \\
\hline Chronic Pulmonary Disease & 1 \\
\hline Chronic Liver Disease & 1 \\
\hline Cerebrovascular Disease & 1 \\
\hline Chronic Neurologic Disease & 1 \\
\hline \multicolumn{2}{|l|}{ Symptom Risk Factor } \\
\hline Dyspnea & 1 \\
\hline
\end{tabular}

https://doi.org/10.1371/journal.pone.0261508.t006

only cohort (OR 2.1 vs 3.5 ), and the odds of immunosuppressed patients without palliative goals of care being admitted were greater (OR 7.0 vs 3.9).

Criteria included in the probabilistic, simplified clinical prediction score are displayed in Table 6. Because cumulative comorbidity count was significantly associated with poor outcomes, we included comorbidities in the simplified tool that were not individually associated with increased risk in the expanded logistic regression model. In the derivation cohort, the AUROC for the simplified clinical prediction score for 14-day hospitalization was 0.82 (95\% CI: 0.81-0.84) and 0.8 (95\% CI: 0.78-0.82) in the validation cohort. AUROC for 28-day allcause mortality in the derivation cohort was 0.91 (95\% CI: 0.83-0.94) and in the hold-out validation cohort 0.80 (95\% CI: 0.69-0.9). In the temporally-independent validation cohort, AUROC for hospitalization was 0.76 (95\% CI 0.75-0.77) and for mortality 0.9 (95\% CI $0.88-$ 0.91 ). The scoring threshold that optimized sensitivity and specificity (by Youden's index [15]) was 6 with test characteristics of $71.1 \%$ and $76.2 \%$ respectively (Table 7). By comparison, in the derivation cohort, the AUROC for Charlson comorbidity index for predicting hospitalization was 0.74 (95\% CI 0.72-0.77) and for mortality 0.82 (95\% CI 0.69-0.95).

\section{Discussion}

Given recent straining hospital volumes and the emergence of promising but limited-availability outpatient therapies for COVID-19, methods are needed to identify patients with COVID19 at highest risk of progression to severe disease, hospitalization and death. Here we describe 
Table 7. Risk Score test characteristics across thresholds.

\begin{tabular}{|c|c|c|c|c|c|}
\hline Point Threshold & Sensitivity & Specificity & PPV & NPV & \% of Positives \\
\hline 3 & $95.0 \%$ & $28.5 \%$ & $7.5 \%$ & $98.9 \%$ & $72.8 \%$ \\
\hline 4 & $89.1 \%$ & $45.7 \%$ & $9.3 \%$ & $98.5 \%$ & $56.3 \%$ \\
\hline 5 & $80.6 \%$ & $62.8 \%$ & $12.1 \%$ & $98.1 \%$ & $39.8 \%$ \\
\hline 6 & $71.1 \%$ & $76.2 \%$ & $16.6 \%$ & $97.5 \%$ & $26.7 \%$ \\
\hline 7 & $60.9 \%$ & $84.1 \%$ & $20.6 \%$ & $97.0 \%$ & $18.7 \%$ \\
\hline 8 & $51.4 \%$ & $89.2 \%$ & $24.4 \%$ & $96.4 \%$ & $13.4 \%$ \\
\hline 9 & $41.4 \%$ & $92.8 \%$ & $28.2 \%$ & $95.9 \%$ & $9.4 \%$ \\
\hline 10 & $32.3 \%$ & $95.2 \%$ & $31.7 \%$ & $95.4 \%$ & $6.5 \%$ \\
\hline 11 & $25.0 \%$ & $97.0 \%$ & $36.1 \%$ & $94.9 \%$ & $4.4 \%$ \\
\hline 12 & $17.4 \%$ & $98.1 \%$ & $38.5 \%$ & $94.6 \%$ & $2.9 \%$ \\
\hline
\end{tabular}

https://doi.org/10.1371/journal.pone.0261508.t007

a simple scoring model capable of accurately risk stratifying ambulatory and emergency department patients for COVID-19 for subsequent hospitalization and mortality.

One of the primary strengths of this model is the simplified and easily calculable score using features that are widely accessible. In particular, our score does not require laboratory studies, which are unavailable in the majority of ambulatory patients testing positive for SARS-CoV-2. While preserving discriminative value, this simple scoring system has potential to facilitate more widespread clinical application in settings lacking robust integration of informatics. The model was derived and validated in a very large and diverse population in the western United States and is based on risk factors for severe disease that are largely conserved across global populations, including age, male sex, overall comorbid burden, and shortness of breath at the time of risk stratification. These factors align closely with those included in models derived in other locations and populations $[3,5-9,16,17]$.

For comparison, the Jehi model [6] was derived and validated in a cohort of 4536 patients in Ohio, USA, and included age, gender, race/ethnicity, income and housing density, smoking status, symptoms, a small set of comorbidities (obesity, asthma, diabetes, hypertension and immunosuppression), as well as laboratory data if available. In the internal validation cohort, AUROC for predicting hospitalization for this model was 0.81 . The Wollenstein-Betech model [8] used data from 91,000 Mexican patients, and demonstrated and AUROC of 0.62 using age, gender, chronic renal insufficiency, diabetes, immunosuppression, COPD, obesity, hypertension, tobacco use, cardiovascular disease and asthma. The Dashti model [5] was derived and internally validated in a cohort of more than 12,000 patients in Massachusetts, USA. Using age, gender, race/ethnicity, smoking status and median household income, this score had an AUROC for hospitalization risk of 0.77 . Finally, we also compared the performance of the Charlson comorbidity index [10] in our own data and found that it was not quite as discriminative for hospitalization, but equally accurate at predicting mortality.

Although race and ethnicity are often omitted from clinical prediction models to prevent illegal or unethical profiling behavior, the National Quality Forum recommended that when applications of risk prediction include patient selection for preventive or therapeutic modalities, omission of race or ethnicity can actually cause inequity in healthcare access and worsen outcomes disparity by underestimating risk using other demographic and clinical features alone [18]. In COVID-19, it is now well-recognized that significant outcome differences among communities of color exist with respect to severe illness and hospitalization [19] despite adjustment for age, gender and underlying medical conditions [5,6]. This remains poorly understood and may be due to social determinants of health, inadequate access to healthcare, or poorly-controlled co-morbidities. Because we anticipated application of this risk 
stratification model to aid in allocating preventive therapies in COVID, we, like other published models [5,6], chose to include race and ethnicity in our score. In future work, more refined socioeconomic, cultural and healthcare access surrogates would be preferable alternatives.

When emergency use authorization (EUA) was granted by the United States Food and Drug Administration for monoclonal antibodies bamlanivimab and casirivimab/imdevimab for administration in non-hospitalized patients with early mild-moderate COVID-19, most states were experiencing peak community transmission, with thousands of new patients per day. It became clear that not only would the supply of drugs be inadequate initially to treat all patients qualifying under EUA criteria, but the capacity to administer infusions without compromising infection control in infusion sites would be even more limited. To address this limited resource situation, the Utah Crisis Standards of Care scarce medications committee was convened with the goal of equitably and efficiently matching available infusion capacity to patients at highest probability of hospitalization most likely to benefit. The simple scoring tool described herein was ultimately adopted because of the simplicity, widely accessible clinical features and validation in a large, representative local population. By regularly adjusting the eligibility criteria based on the risk score threshold that best calibrates current infusion capacity to the number of new cases in high-risk strata, this risk-targeted drug allocation strategy has provided an equitable and flexible means of drug delivery in the context of still-uncertain efficacy and limited resources.

Limitations of our study include the retrospective, observational design, and the possibility that comorbidity data may have been unavailable or out of date for some patients in the cohort who receive the majority of their medical care outside our integrated healthcare system.

Although the large study population and inclusion of widely recognized features improves the likelihood of generalizability, this will need to be confirmed through external validation before adoption in other populations.

\section{Conclusion}

In this large retrospective cohort study, we identified simple risk factors that can easily be calculated at the bedside without laboratory values to risk stratify COVID-positive individuals for risk of hospitalization and death. Applications include guiding allocation of therapies that are limited in availability. External validation is needed to confirm generalizability in diverse and geographically independent population.

\section{Supporting information}

S1 File.

(XLSX)

\section{Author Contributions}

Conceptualization: Brandon J. Webb, Nancy Grisel, Samuel M. Brown, Ithan D. Peltan, Emily S. Spivak, Joseph Bledsoe.

Data curation: Brandon J. Webb, Nancy Grisel.

Formal analysis: Brandon J. Webb, Samuel M. Brown, Ithan D. Peltan, Joseph Bledsoe.

Investigation: Brandon J. Webb.

Methodology: Brandon J. Webb, Samuel M. Brown, Ithan D. Peltan, Joseph Bledsoe. 
Project administration: Brandon J. Webb.

Supervision: Brandon J. Webb.

Writing - original draft: Brandon J. Webb, Nicholas M. Levin, Nancy Grisel, Samuel M. Brown, Ithan D. Peltan, Emily S. Spivak, Mark Shah, Eddie Stenehjem, Joseph Bledsoe.

Writing - review \& editing: Brandon J. Webb, Nicholas M. Levin, Nancy Grisel, Samuel M. Brown, Ithan D. Peltan, Emily S. Spivak, Mark Shah, Eddie Stenehjem, Joseph Bledsoe.

\section{References}

1. Zhu N, Zhang D, Wang W, Li X, Yang B, Song J, et al. A Novel Coronavirus from Patients with Pneumonia in China, 2019. New England Journal of Medicine. 2020 Feb 20; 382(8):727-33.

2. Wang D, Hu B, Hu C, Zhu F, Liu X, Zhang J, et al. Clinical Characteristics of 138 Hospitalized Patients With 2019 Novel Coronavirus-Infected Pneumonia in Wuhan, China. JAMA. 2020 Mar 17; 323 (11):1061. https://doi.org/10.1001/jama.2020.1585 PMID: 32031570

3. Wollenstein-Betech S, Silva AAB, Fleck JL, Cassandras CG, Paschalidis IC. Physiological and socioeconomic characteristics predict COVID-19 mortality and resource utilization in Brazil. PLOS ONE. 2020 Oct 14; 15(10):e0240346. https://doi.org/10.1371/journal.pone.0240346 PMID: 33052960

4. Pastor-Barriuso R, Pérez-Gómez B, Hernán MA, Pérez-Olmeda M, Yotti R, Oteo-Iglesias J, et al. Infection fatality risk for SARS-CoV-2 in community dwelling population of Spain: nationwide seroepidemiological study. BMJ [Internet]. 2020 Nov 27 [cited 2020 Dec 1];371. Available from: https://www.bmj.com/ content/371/bmj.m4509. https://doi.org/10.1136/bmj.m4509 PMID: 33246972

5. Dashti H, Roche EC, Bates DW, Mora S, Demler O. SARS2 simplified scores to estimate risk of hospitalization and death among patients with COVID-19. medRxiv [Internet]. 2020 Sep 13 [cited 2020 Nov 22]; Available from: https://www.ncbi.nlm.nih.gov/pmc/articles/PMC7491527/. https://doi.org/10.1101/ 2020.09.11.20190520 PMID: 32935112

6. Jehi L, Ji X, Milinovich A, Erzurum S, Merlino A, Gordon S, et al. Development and validation of a model for individualized prediction of hospitalization risk in 4,536 patients with COVID-19. PLOS ONE. 2020 Aug 11; 15(8):e0237419. https://doi.org/10.1371/journal.pone.0237419 PMID: 32780765

7. Fisman DN, Greer AL, Hillmer M, Tuite AR. Derivation and Validation of Clinical Prediction Rules for COVID-19 Mortality in Ontario, Canada. Open Forum Infect Dis [Internet]. 2020 Oct 5 [cited 2020 Nov 30]; Available from: https://www.ncbi.nlm.nih.gov/pmc/articles/PMC7650986/. https://doi.org/10.1093/ ofid/ofaa463 PMID: 33204755

8. Wollenstein-Betech S, Cassandras CG, Paschalidis ICh. Personalized predictive models for symptomatic COVID-19 patients using basic preconditions: Hospitalizations, mortality, and the need for an ICU or ventilator. Int J Med Inform. 2020 Oct; 142:104258. https://doi.org/10.1016/j.jimedinf.2020.104258 PMID: 32927229

9. Zhang C, Qin L, Li K, Wang Q, Zhao Y, Xu B, et al. A Novel Scoring System for Prediction of Disease Severity in COVID-19. Front Cell Infect Microbiol [Internet]. 2020 [cited 2020 Dec 5];10. Available from: https://www.frontiersin.org/articles/10.3389/fcimb.2020.00318/full. https://doi.org/10.3389/fcimb.2020. 00010 PMID: 32117794

10. Deyo RA, Cherkin DC, Ciol MA. Adapting a clinical comorbidity index for use with ICD-9-CM administrative databases. J Clin Epidemiol. 1992 Jun; 45(6):613-9. https://doi.org/10.1016/0895-4356(92)901338 PMID: 1607900

11. Elixhauser A, Steiner C, Harris DR, Coffey RM. Comorbidity measures for use with administrative data. Med Care. 1998 Jan; 36(1):8-27. https://doi.org/10.1097/00005650-199801000-00004 PMID: 9431328

12. Defining Adult Overweight and Obesity | Overweight \& Obesity | CDC [Internet]. 2020 [cited 2021 Feb 16]. Available from: https://www.cdc.gov/obesity/adult/defining.html.

13. NAGELKERKE NJD. A note on a general definition of the coefficient of determination. Biometrika. 1991 Sep 1; 78(3):691-2.

14. Maddala GS. Limited-Dependent and Qualitative Variables in Econometrics [Internet]. Cambridge: Cambridge University Press; 1983 [cited 2021 Feb 16]. (Econometric Society Monographs). Available from: https://www.cambridge.org/core/books/limiteddependent-and-qualitative-variables-ineconometrics/69B8DBC75160713AA3AD1AD979D297B8.

15. Youden WJ. Index for rating diagnostic tests. Cancer. 1950 Jan; 3(1):32-5. https://doi.org/10.1002/ 1097-0142(1950)3:1<32::aid-cncr2820030106>3.0.c0;2-3 PMID: 15405679

16. Mesas AE, Cavero-Redondo I, Álvarez-Bueno C, Sarriá Cabrera MA, Maffei de Andrade S, SequíDominguez I, et al. Predictors of in-hospital COVID-19 mortality: A comprehensive systematic review 
and meta-analysis exploring differences by age, sex and health conditions. PLoS One. 2020; 15(11): e0241742. https://doi.org/10.1371/journal.pone.0241742 PMID: 33141836

17. de Terwangne C, Laouni J, Jouffe L, Lechien JR, Bouillon V, Place S, et al. Predictive Accuracy of COVID-19 World Health Organization (WHO) Severity Classification and Comparison with a BayesianMethod-Based Severity Score (EPI-SCORE). Pathogens. 2020 Oct 24; 9(11).

18. NQF: Risk Adjustment for Socioeconomic Status or Other Sociodemographic Factors [Internet]. [cited 2020 Nov 30]. Available from: https://www.qualityforum.org/Publications/2014/08/Risk_Adjustment_ for_Socioeconomic_Status_or_Other_Sociodemographic_Factors.aspx.

19. CDC. Coronavirus Disease 2019 (COVID-19) [Internet]. Centers for Disease Control and Prevention. 2020 [cited 2020 Nov 30]. Available from: http://www.cdc.gov/coronavirus/2019-ncov/covid-data/ investigations-discovery/hospitalization-death-by-race-ethnicity.html. 